\title{
A Formal Solution for Slit Corrections in Small-Angle X-Ray Scattering
}

\author{
Jacob Mazur and Andrew M. Wims \\ Institute for Materials Research, National Bureau of Standards, Washington, D.C., 20234
}

(May 5, 1966)

\begin{abstract}
Slit shaped apertures are usually used in small-angle $\mathrm{x}$-ray scattering measurements in order to obtain easily measured intensities of scattered radiation. As a result, the scattering intensity at a given angle determined by the camera, the center of the sample, and the central incident x-ray beam is not simply related to the scattering from the sample only at that angle. The experimentally determined intensities $\tilde{I}(x)$ are related to the true scattering intensities by the following integral equation:

$$
\tilde{I}(x)=\int_{-\infty}^{\infty} I\left[\left(x^{2}+t^{2}\right)^{1 / 2}\right] W(t) d t .
$$

This integral equation has been previously solved only for certain simplified functional forms for $W(t)$ and $\tilde{I}(x)$. In this paper, a formal procedure is developed for calculating $I(x)$ from the observed angular measurements, which does not necessitate making any a priori assumption about the form of $W(t)$ and $\tilde{I}(x)$.
\end{abstract}

Key words: Distribution of intensity, integral equation, scattering cross section, slit correction, small-angle $\mathrm{x}$-ray scattering.

\section{Introduction}

Narrow slits are often used in small-angle x-ray scattering measurements to collimate the incident beam sufficiently to allow scattering measurements extremely close to the incident beam. Compared to a pinhole of diameter approximately equal to the slit width, the slit system increases the available scattering energy. However, the scattered radiation measured in a given solid angle can no longer be simply described as the scattering coming from a point source. Instead, the actual angular scattering measurements, which are summations of the scattering contributed by each scattering center along the height of the sample slit, must be resolved to give the scattering of an infi- nitely small area at the center of the sample slit. In this paper, a formal procedure is described for calculating the true scattering cross sections from the observed angular measurements when long but very narrowwidth slits are used for collimation.

A diagram for the slit system of a small angle x-ray system is given in figure 1 . This is a modification of the diagram given by Shull and Roess [1]. ${ }^{1}$ The plane of the quadrangle $t x y r$ is perpendicular to the camera axis which is in the vertical plane containing the collimating slit and the sample slit. The collimating slit nearest the x-ray tube has length $2 l_{1}$, the effective length of the slit at the sample is $2 l_{2}$, and the

${ }^{1}$ Figures in brackets indicate the literature references at the end of this paper.

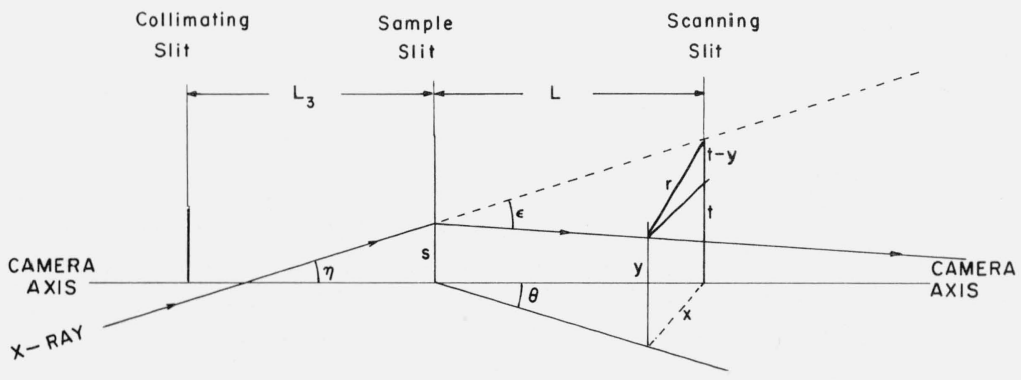

Figure 1. Diagram of a slit collimation system. 
scanning slit at the detector is of length $2 y_{0}$. The scanning slit and sample slit are shown in figure 1 to be in a plane making an angle $\theta$ with the plane containing the camera axis and the collimating slit. Let us consider a ray which makes an angle $\eta$ with the camera axis and after it hits the sample is scattered by an angle $\epsilon$ with its original direction. Since the angles $\epsilon, \theta$, and $\eta$ are in practice small, they can be replaced by the following ratios:

$$
\begin{aligned}
& \epsilon=r / L, \\
& \theta=x / L,
\end{aligned}
$$

and $\eta=(t-s) / L$.

Since $r^{2}=x^{2}+(t-y)^{2}$, it follows that

$$
\epsilon^{2}=\theta^{2}+\left[\eta+\left(\frac{s-y}{L}\right)\right]^{2}
$$

Let $\tilde{I}(\theta)$ be the intensity striking the scanning slit centered at $\theta$, and let $I(\epsilon) d \eta$ represent the increment in intensity of scattering in an element $d \eta$. The following equation defines $\tilde{I}(\theta)$ by averaging $I(\epsilon)$ over the entire scanning area.

$$
\tilde{I}(\theta)=\frac{1}{2 y_{0}} \int_{-y_{0}}^{y_{0}} d y \int_{-l 2}^{l_{2}} d s \int_{\left(s-l_{1}\right) / L_{3}}^{\left(s+l_{1}\right) / L_{3}} I(\epsilon) d \eta .
$$

Equation (3) is the one obtained by Shull and Roess [1].

The triple integral in eq (3) can be reduced to a single integral. One way to do it is by the method employed by Schmidt [2]. By changing $y, s$, and $\eta$ into the dimensionless variables

$$
y_{1}=y / L_{1}, s_{1}=s\left(\frac{1}{L_{3}}+\frac{1}{L}\right), \eta_{1}=\eta-\frac{s}{L_{3}}
$$

the limits of the integrations are replaced by constants which are descriptive of the geometrical parameters of the slit system. One can then integrate the resulting expression over $s_{1}$ and $y_{1}$. After substituting $t_{1}=\frac{t}{L}$ for $\eta_{1}+s_{1}$, the resulting integral equation is

$$
\tilde{I}(\theta)=A \int_{0}^{\infty} I\left[\left(\theta^{2}+t_{1}^{2}\right)^{1 / 2}\right] W\left(t_{1}\right) d t_{1} .
$$

However, $W\left(t_{1}\right)$ is a complicated function of the geometrical parameters of the system. In general, $W\left(t_{1}\right)$ is a quadratic function of $t_{1}$, represented by different functions for different intervals of $t_{1}$. Also, $W\left(t_{1}\right)$ must be premultiplied by a unit step function which becomes zero when

$$
t>\left|y_{0}+l_{2}+\left(l_{1}+l_{2}\right) \frac{L}{L_{3}}\right| .
$$

The advantage of starting with eq (3) is that $L_{3} \eta_{1}$ is the coordinate of the length of the slit nearest to the x-ray tube. Thus, in the more general case when the intensity along $\eta_{1}$ is not uniform, but is given by a general distribution function $g\left(\eta_{1}\right)$, eq (4) will still be obtained. However, $W\left(t_{1}\right)$ will be represented by double integrals of the general form

$$
\iint g\left(\eta_{1}-s_{1}-y_{1}\right) d s_{1} d y_{1}
$$

with the ranges of integration being determined by the parameters of the system. In other words, $W\left(t_{1}\right)$ in eq (4) results from the projections of the variables $s_{1}$ and $y_{1}$ onto the plane of registration, corrected for a possible nonuniformity in the distribution of intensity of scattered ratiation along $\eta_{1}$. The only restrictions imposed are on the variables $s_{1}, \eta_{1}$, and $y_{1}$. These angles must be sufficiently small to justify their replacements by ratios of coordinates (eq (1)).

In the process of determining the formal solution of the integral eq (4), one has to calculate derivatives of $W(t)$, and for this reason, it would be more advantageous to start with a continuous expression for this function. This difficulty is eliminated if one starts with the experimentally determined $W(t)$ or by using the smoothed curve obtained from the calculated segments.

Starting with eq (3), but omitting the slit coordinate $\eta_{1}$, we have the following equation, with $w(t)$ representing the intensity distribution in the $t$ direction

$$
\tilde{I}(x)=\int_{-\infty}^{\infty} \int_{-\infty}^{\infty} w(t) I\left\{\left[x^{2}+(t-y)^{2}\right]^{1 / 2}\right\} d t d y .
$$

Interchanging the order of integration and making use of the fact that $W(t)=W(-t)$, we obtain

$$
\tilde{I}(x)=2 \int_{0}^{\infty} W(t) I\left[\left(x^{2}+t^{2}\right)^{1 / 2}\right] d t
$$

with

$$
W(t)=\int_{-\infty}^{\infty} w(t-y) d y .
$$

$W(t)$ can be determined by measuring the intensity in the plane of registration. This is done by taking various intervals equal to $2 y_{0}$ along the $t$ axis. These intervals are equal to the length of the scanning slit. The finite length of the scanning slit restricts the range of the variable $y$ in eq (7). Therefore, a unit step function $h(y)$ has to be introduced, with $h(y)=0$ for $y>k$ and $h(y)=1$ for $0<y<k$. This process will modify $W(t)$ into a convolution integral of $w(t)$ and $h(t)$,

$$
W(t)=\int_{-\infty}^{\infty} w(y) h(t-y) d y .
$$

The only known solutions of the integral eq (6) were obtained for certain special forms of $W(t)$ and of $\tilde{I}(x)$. Guinier [3], Fournet [4], and DuMond [5] have solved this integral equation for slits of infinite height $(W(t)=1)$. Their method was extended by Kratky, Porod, and Kahovec [6], and by Gerold [7] to finite beams where $W(t)$ could be represented by a step function, or a Gaussian distribution, or a particular trapezoidal distribution. Shull and Roess [1] have 
presented a solution for an arbitrary $W(t)$ if the scattering curve $\tilde{I}(x)$ can be resolved into a packet of Gaussian functions. The earlier attempts to solve eq (6) have been recently reviewed by Beeman, Kaesberg, Anderegg, and Webb [8], and by Kratky, Porod, and Skala [9].

In the following section a formal solution to eq (6) is presented which does not require any a priori assumptions about the particular forms of $\tilde{I}(x)$ and of $W(t)$.

\section{Solution of the Integral Equation}

\subsection{General Considerations and Discussions}

Equation (6) is rewritten as

$$
\tilde{I}(x)=2 \int_{x}^{\infty} \frac{y I(y) W\left[\left(y^{2}-x^{2}\right)^{1 / 2}\right] d y}{\left(y^{2}-x^{2}\right)^{1 / 2}} .
$$

To solve this integral equation in one of the standard forms it is essential to remove its singularity, since the kernel of the equation is infinite at $y=x$. The transformation of this equation, which removes this singularity follows the method employed in connection with the celebrated Abel's integral equation. A new variable in a suitable form is introduced, and both sides of the resulting integral equation are integrated over the variable $x$ between the value of the new variable and infinity. This, combined with the interchange in the order of integration of the right-hand side of the resulting integral equation, through the use of the Dirichlet formula, has the effect of removing the singularity. The new kernel is finite everywhere, but it is replaced by a bounded integral whose integrand involves the original distribution function $W(t)$.

The solution thus obtained is an implicit one, since the left-hand side of the integral equation involves the integrated form of the experimental function $\tilde{I}(x)$. In order to obtain an explicit form for the integral equation, two additional steps are required: (1) Solution of a subsidiary integral equation for another function, which essentially compensates for the slit weighting function $W$, (see following section), and (2) differentiation of the experimental intensity function $\tilde{I}(x)$ with respect to $x$. The details of the solution are given in the following section. Here we give the two solutions, the implicit and the explicit one in their final forms: Implicit Solution:

$$
\int_{0}^{\infty} \tilde{I}\left[\left(\nu^{2}+u^{2}\right)^{1 / 2}\right] d \nu=2 \int_{u}^{\infty} y I(y) K\left[\left(y^{2}-u^{2}\right)^{1 / 2}\right] d y,
$$

with

$$
K(\nu)=\int_{0}^{1} \frac{W(\nu x)}{\left(1-x^{2}\right)^{1 / 2}} d x ; \quad \nu^{2}=y^{2}-u^{2}
$$

Explicit Solution:

$$
I(u)=\frac{1}{\pi} \int_{0}^{\infty} \frac{\left.\tilde{I}\left(y^{2}+u^{2}\right)^{1 / 2}\right]}{\left(y^{2}+u^{2}\right)^{1 / 2}} F(y) d y .
$$

The function $F$ satisfies the following Volterra equation of the second kind:

$$
F(y)=1-\frac{2}{\pi} y \int_{0}^{y} F(z) K\left[\left(y^{2}-z^{2}\right)^{1 / 2}\right] d z,
$$

$$
K(\nu)=\frac{l}{\nu} \int_{0}^{1} \frac{x W^{\prime}(\nu x) d x}{\left(1-x^{2}\right)^{1 / 2}} ; \quad \nu^{2}=y^{2}-z^{2}
$$

The kernels of the implicit and explicit solutions, eqs (10a) and (12a), are similar in their general forms, except that the first one involves the slit correction function $W$, and the second one involves its derivative. The implicit solution is suitable only with the use of a digital computer. Both sides of eq (10) are solved simultaneously as a set of simultaneous linear equations through a matrix inversion method. This method can be employed also in order to obtain $F(u)$ from eq (12). However, the latter equation can be solved by expansion of $F(u)$ in a Liouville-Neumann series. These series will converge rapidly, if a good initial assumption is made about the approximate form of the function $F(u)$.

Summarizing, one can demonstrate that the solution to the problem of slit correction always leads to a single integral equation provided that all angular variables of the x-ray camera-slit system are small. The solution of this integral equation invariably leads to a solution of the Volterra equation of the second kind which has for its inhomogeneous part a constant term, and whose kernel involves an integrated expression of the derivative of the experimentally determinable slit correction function.

At the present time a computer program for the numerical solution of the eqs $(10-12)$ is being developed.

\subsection{Derivation of Equations (10-12)}

In Abel's integral equation, the kernel $K(x, y)$ is of the form $\frac{1}{(x-y)^{\alpha}}$, with $0<\alpha<1$ and $x>y$. For $\alpha=1 / 2$, the singularity of the kernel is removed by multiplication of both sides of the integral equation by $\frac{d x}{(u-x)^{1 / 2}}$ and integrating with respect to $x$. This is done to make use of the fact that

$$
\int_{y}^{u} \frac{d x}{[(x-y)(u-x)]^{1 / 2}}=\pi .
$$

With $W\left[\left(y^{2}-x^{2}\right)^{1 / 2}\right]=K(x, y)$, eq (6) is written in 
Abelian form:

$$
\tilde{I}(x)=2 \int_{x}^{\infty} \frac{y I(y) K(x, y)}{\left(y^{2}-x^{2}\right)^{1 / 2}} d y .
$$

For this particular equation, the transformation which removes the singularity is based not on eq (13), but on an equivalent form: Use is made of the fact, that

$$
\int_{u}^{y} \frac{x d x}{\left[\left(y^{2}-x^{2}\right)\left(x^{2}-u^{2}\right)\right]^{1 / 2}}=\frac{\pi}{2},
$$

since eq (15) can be shown to reduce to eq (13). We therefore multiply both sides of eq (14) by

$$
\frac{x d x}{\left(\mathrm{x}^{2}-u^{2}\right)^{1 / 2}}
$$

and integrate over $x$ from $u$ to $\infty$, obtaining

$$
\begin{gathered}
\int_{u}^{\infty} \frac{x \tilde{I}(x) d x}{\left(x^{2}-u^{2}\right)^{1 / 2}}=2 \int_{u}^{\infty} \int_{x}^{\infty} \frac{x y I(y) W\left[\left(y^{2}-x^{2}\right)^{1 / 2} d y d x\right.}{\left[\left(y^{2}-x^{2}\right)\left(x^{2}-u^{2}\right)\right]^{1 / 2}} \\
=2 \int_{u}^{\infty} y I(y) K_{1}(u, y) d y,
\end{gathered}
$$

with

$$
K_{1}(u, y)=\int_{u}^{y} \frac{\left.x W\left[y^{2}-x^{2}\right)^{1 / 2}\right] d x}{\left[\left(x^{2}-u^{2}\right)\left(y^{2}-x^{2}\right)\right]^{1 / 2}}
$$

In obtaining eq (16) and (17), the order of integration was exchanged using the following Dirichlet formula:

$$
\int_{u}^{\infty} d x \int_{x}^{\infty} G(y, x) d y=\int_{u}^{\infty} d y \int_{u}^{y} G(y, x) d x .
$$

The implicit solution (eq (10-10a)) is obtained from (16) and (17) in a straightforward way: we replace $y^{2}-x^{2}$ by $t^{2}$. The kernel (17) simplifies to

$$
K_{1}(u, y)=\int_{0}^{\left(y^{2}-u^{2}\right)^{1 / 2}} \frac{W(t) d t}{\left(y^{2}-u^{2}-t^{2}\right)^{1 / 2}},
$$

and eq (10-10a) follow directly. To obtain the explicit solution of eq (14), we multiply the integrands of both sides of eq (16) by $F\left[\left(x^{2}-u^{2}\right)^{1 / 2}\right] . \quad F(z)$ is an unknown function to be determined subsequently. In the right-hand side of eq $(16) K_{2}(u, y)$ replaces $K_{1}(u, v)$ and is given by eq (20):

$$
K_{2}(u, y)=\int_{u}^{y} \frac{x F\left[\left(x^{2}-u^{2}\right)^{1 / 2}\right] W\left[\left(y^{2}-x^{2}\right)^{1 / 2}\right] d x}{\left[\left(y^{2}-x^{2}\right)\left(x^{2}-u^{2}\right)\right]^{1 / 2}} .
$$

Both sides of the resulting eq (16) are now differentiated with respect to $u$ :

$$
\begin{aligned}
\frac{d}{d u} \int_{u}^{\infty} \frac{x \widetilde{I}(x) F\left[\left(x^{2}-u^{2}\right)^{1 / 2}\right]}{\left(x^{2}-u^{2}\right)^{1 / 2}} d x \\
\quad=-2 u I(u) K_{2}(u, u)+2 \int_{u}^{\infty} y I(y) \frac{d K_{2}(u, y)}{d u} d y .
\end{aligned}
$$

We need a function $F(z),\left(z^{2}=x^{2}-u^{2}\right)$, which leads to $K_{2}(u, y)=\lambda$, independent of $u$ and $y$. If $K_{1}(x, y)$ is constant, $F(z)$ then would be a constant alse, equal to $2 / \pi$. We would then have an Abel-related integral quation with a well-known solution for $I(x)$. With $K_{2}(u, y)=\lambda$, the solution of eq $(21)$ is readily obtained:

$$
I(u)=-\frac{1}{2 u \lambda} \frac{d}{d u} \int_{0}^{\infty} \tilde{I}\left[\left(z^{2}+u^{2}\right)^{1 / 2}\right] F(z) d z,
$$

or

$$
I(u)=-\frac{1}{2 \lambda} \int_{0}^{\infty} \frac{\tilde{I}\left[\left(z^{2}+u^{2}\right)^{1 / 2}\right]}{\left(z^{2}+u^{2}\right)^{1 / 2}} F(z) d z .
$$

Equation (23) was obtained by Kratky, Porod, and Kahovec [6], using a different method of derivation.

At first glance, the difficulty we encountered in solving the original integral eq (14), which is brought on by the singularity of its kernel, does not seem to be removed. The integral equation for the new function $F(z)$ still possesses a similar singularity. However, the integral equation for $F(z)$ (eq (20)) is considerably simpler than the original integral equation for $I(x)$ if $K_{2}(u, y)=$ constant. The same procedures which were used to remove the singularity in the kernel of eq (14) lead to a straight-forward solution for $F(z)$ by reducing it to a Volterra-type equation of the second kind. Equation (20) is rewritten as

$$
\int_{0}^{\left(y^{2}-u^{2}\right)^{1 / 2}} \frac{F(z) W\left[\left(y^{2}-u^{2}-z^{2}\right)^{1 / 2}\right]}{\left(y^{2}-u^{2}-z^{2}\right)^{1 / 2}} d z=\lambda,
$$

or

$$
\int_{0}^{s} \frac{F(z) W\left[\left(s^{2}-z^{2}\right)^{1 / 2}\right]}{\left(s^{2}-z^{2}\right)^{1 / 2}} d z=\lambda
$$

with $s^{2}=y^{2}-u^{2}$.

Both sides of eq (24) are then multiplied by $s\left(u^{2}-s^{2}\right)^{-1 / 2}$ and integrated over $s$ from $o$ to $u$. The following equation is obtained:

$$
\int_{0}^{u} \int_{0}^{s} \frac{s F(z) W\left[\left(s^{2}-z^{2}\right)^{1 / 2}\right] d z d s}{\left[\left(s^{2}-z^{2}\right)\left(u^{2}-s^{2}\right)\right]^{1 / 2}}=\lambda \int_{0}^{u} \frac{s d s}{\left(u^{2}-s^{2}\right)^{1 / 2}}=\lambda u .
$$

We again interchange the order of integration in the l.h.s of eq (25), using the Dirichlet formula to obtain

$$
\int_{0}^{u} F(z) d z \int_{z}^{u} \frac{s W\left[\left(s^{2}-z^{2}\right)^{1 / 2} d s\right.}{\left[\left(s^{2}-z^{2}\right)\left(u^{2}-s^{2}\right)\right]^{1 / 2}}=\lambda u .
$$

Next we subtract and add $W(z=s)=W(0)$ and integrate eq (26) to obtain

$$
\begin{aligned}
& \frac{\pi}{2} W(0) \int_{0}^{u} F(z) d z-\int_{0}^{u} F(z) M(u, z) d z=\lambda u, \\
& M(u, z)=\int_{z}^{u} \frac{s\left\{W(0)-W\left[\left(s^{2}-z^{2}\right)^{1 / 2}\right]\right\}}{\left[\left(s^{2}-z^{2}\right)\left(u^{2}-s^{2}\right)\right]^{1 / 2}} d s .
\end{aligned}
$$


After differentiating (27) with respect to $u$ we obtain the following Volterra-type equation of the second kind

$$
F(u)=\frac{2 \lambda}{\pi W(0)}+\frac{2}{\pi W(0)} \int_{0}^{u} \frac{d M(u, z)}{d u} F(z) d z
$$

since $M(u, u)=0$.

Equation (28) can also be further simplified. If we set $W(0)=1$ and $\lambda=\pi / 2$, then

$$
F(u)=1+\frac{2}{\pi} \int_{0}^{u} \frac{d M(u, z)}{d u} F(z) d z .
$$

Equation (27a) is integrated by parts,

$$
\begin{aligned}
M(u, z)= & \frac{\pi}{2}\left[1-W\left[\left(u^{2}-z^{2}\right)^{1 / 2}\right]\right. \\
& +\int_{z}^{u} \frac{\left.s W^{\prime}\left[s^{2}-z^{2}\right)^{1 / 2}\right]}{\left(s^{2}-z^{2}\right)^{1 / 2}} \cdot \sin ^{-1}\left[\left(\frac{s^{2}-z^{2}}{u^{2}-z^{2}}\right)^{1 / 2}\right] d s .
\end{aligned}
$$

Therefore,

$$
\frac{d M(u, z)}{d u}=-\frac{u}{\left(u^{2}-z^{2}\right)} \int_{z}^{u} \frac{s W^{\prime}\left[\left(s^{2}-z^{2}\right)^{1 / 2}\right]}{\left[\left(u^{2}-s^{2}\right)\right]^{1 / 2}} d s .
$$

Integral eq (29) assumes the following form

$$
F(u)=1-\frac{2}{\pi} \int_{0}^{u} F(z) K(u, z) d z,
$$

with

$$
K(u, z)=\frac{u}{\left(u^{2}-z^{2}\right)} \int_{0}^{\left(u^{2}-z^{2}\right)^{1 / 2}} \frac{y W^{\prime}(y) d y}{\left[\left(u^{2}-z^{2}-y^{2}\right)\right]^{1 / 2}} .
$$

The kernel of eq (31) is finite for all values of $z$, including $z=u$.

Equations (12-12a) follow directly by exchanging variables, $u$ being replaced by $y$.

\section{References}

[1] C. G. Shull and L. C. Roess, J. Appl. Phys. 18, 295 (1947).

[2] P. Schmidt (private communication).

[3] A. Guinier and G. Fournet, J. Phys. Radium 8, 345 (1947a).

[4] A. Guinier and G. Fournet, Nature, London 160, 504 (1947b).

[5] J. Du Mond, Phys. Rev. 72, 83 (1947).

[6] O. Kratky, G. Porod, and L. Kahovec, Z. Electrochem. 58, 49 (1951).

[7] V. Gerold, Acta Cryst. 10, 287 (1957).

[8] Beeman, Kaesberg, Anderegg, and Webb, Handbuch Der Physik XXXII, 321 (1957).

[9] O. Kratky, G. Porod, Z. Skala, Acta Phys. Austriaca 13, 76 